\title{
Transit times of interplanetary coronal mass ejections and the solar wind speed
}

\author{
B. Vršnak and T. Žic
}

\author{
Hvar Observatory, Faculty of Geodesy, Kačićeva 26, 10000 Zagreb, Croatia \\ e-mail: bvrsnak@geof.hr
}

Received 17 March 2007 / Accepted 12 June 2007

\begin{abstract}
Aims. The Sun-Earth transit time of interplanetary coronal mass ejections (ICMEs) is one of central issues of space weather forecasting. Our aim is to find out to what degree the ICME transit time depends on the solar wind speed.

Methods. Two samples of coronal mass ejections (CMEs) and the associated ICMEs are used to analyze the relationship between transit times, $T T$, and the solar wind speed, $w$, measured at $1 \mathrm{AU}$ ahead and behind the ICME.

Results. We found a distinct correlation $T T(w)$, clearly showing that the transit time is dependent not only on the ICME take-off speed $v_{\mathrm{CME}}$, but also on the solar wind speed. After dividing the samples into the solar wind speed bins $w \leq 400,400<w \leq 500$, and $w>500 \mathrm{~km} \mathrm{~s}^{-1}$, we compared the corresponding $T T\left(v_{\mathrm{CME}}\right)$ correlations to find that the transit times in the case of $w \leq 400 \mathrm{~km} \mathrm{~s}^{-1}$ subset are longer, on average, for about $20-30 \mathrm{~h}$ than in the case of the $w>500 \mathrm{~km} \mathrm{~s}^{-1}$ subset.

Conclusions. Since the ICME transit time is significantly influenced by the solar wind speed, this effect should be included in statistical and kinematical methods of the space weather forecast.
\end{abstract}

Key words. Sun: coronal mass ejections (CMEs) - Sun: solar-terrestrial relations - Sun: solar wind

\section{Introduction}

Coronal mass ejections (CMEs), i.e., their interplanetary counterparts (interplanetary CMEs; ICMEs) are the main source of major geomagnetic storms (e.g., Gosling et al. 1990; Bothmer \& Schwenn 1995; Tsurutani \& Gonzalez 1998; Zhang et al. 2003; Koskinen \& Huttunen 2006). Consequently, one of the central points of space weather forecasting is prediction of the ICME arrival on Earth, utilizing coronagraphic observations of CMEs. The problem is usually approached with a statistical, kinematical, or MHD-based numerical procedure.

Generally, the statistical method is based on the correlation between the Sun-Earth transit time $T T$ of ICMEs and the CME speed, $v_{\mathrm{CME}}$, measured from coronagraphic observations (hereinafter the ICME take-off speed). This is the simplest forecasting procedure since the correlation straightforwardly provides the most probable transit time directly from the CME speed. However, the $T T\left(v_{\mathrm{CME}}\right)$ correlation is burdened by large scatter of $T T$ values at a given $v_{\mathrm{CME}}$. The overall scatter of data points at a given $v_{\mathrm{CME}}$ usually ranges around \pm 1 day (see the distribution of data points in $T T\left(v_{\mathrm{CME}}\right)$ graphs in, e.g., Gopalswamy et al. 2000; Gopalswamy et al. 2003; Cane \& Richardson 2003a; Cho et al. 2003; Manoharan et al. 2004; Michałek et al. 2004; Srivastava \& Venkatakrishnan 2004; Xie et al. 2006). As emphasized by, e.g., Michałek et al. (2003), Xie et al. (2004), and Schwenn et al. (2005), the prime reason for such a large scatter might be the difference between the measured plane-of-sky speed and the true space velocity. Unfortunately, the problem of projection effects is very complex (Vršnak et al. 2007a), so it is not surprising that application of simple geometrical models does not significantly reduce the scatter of data points in $T T\left(v_{\mathrm{CME}}\right)$ graphs (e.g., compare Figs. $6 \mathrm{a}$ and $\mathrm{b}$ in Gopalswamy et al. 2001; check also Fig. 10 in Schwenn et al. 2005).
The accuracy of the arrival-time predictions can be improved by applying kinematical modeling of the CME propagation, which is based on the fact that ICMEs persistently accelerate/decelerate during their propagation, depending on their speed relative to the solar wind: slow CMEs are accelerated by the solar wind, whereas fast CMEs are decelerated (e.g., Gopalswamy et al. 2001; Vršnak et al. 2004a and references therein). Such behavior indicates that the "aerodynamic" drag plays an essential role in the propagation of ejections (e.g., Vršnak 2001; Cargill 2004). In the simplest form, kinematical models employ an effective constant acceleration whose value depends on the ICME take-off speed (most often taken to be the mean speed of a CME in the coronagraph field of view). The transit time is then calculated by assuming that such an acceleration acts over a certain range of heliocentric distances (e.g., Manoharan et al. 2004; Michałek et al. 2004). This procedure can decrease the transittime error below $\approx 12 \mathrm{~h}$ in a majority of events (e.g., see Fig. 8 in Michałek et al. 2004). In more advanced kinematical models, the transit time is evaluated from the equation of motion in which the acceleration is explicitly expressed in terms of the drag force, i.e., the driving force and gravity are neglected (for the relative contribution of forces see Vršnak et al. 2004a). In other words, the acceleration is a function of the difference between the CME velocity and the solar wind speed (e.g., Vršnak \& Gopalswamy 2002; see also Sect. 5 of this paper).

The numerical approach, based on large-scale MHD models, in the most advanced form includes modeling of the preeruption solar wind through which the ICME has to propagate (e.g., Fry et al. 2003; Manchester et al. 2004; Odstrčil et al. 2005; and references therein). Although this kind of forecasting is certainly the most sophisticated one in a physical sense, the accuracy of the predicted arrival times is still not significantly better than in the case of some kinematical models; compare, 
e.g., the summary of numerical results reported by McKennaLawlor et al. (2006), Dryer et al. (2004), Oler (2004) with the outcome of the kinematical modeling by Michałek et al. (2004) or Vršnak \& Gopalswamy (2002).

In the kinematical drag-based modeling, as well as in the numerical modeling, an important parameter is the solar wind speed. The aim of this paper is to investigate, from an empirical point of view, to what degree the solar wind speed influences the ICME transit time. In combination with predicting the solar wind parameters (Robbins et al. 2006; Vršnak et al. 2007b), the results can be used straightforwardly for statistical forecasting of ICME arrival times, employing the two-parameter dependence of the transit time on the CME coronal speed and the solar wind speed. On the other hand, details of this dependence could give better insight into the characteristics of the drag acceleration or, more precisely, the drag parameter $\gamma$ that represents the inverse deceleration length (for details see Vršnak 2001; Cargill 2004). This could also improve forecasting based on kinematical modeling.

\section{The data set}

\subsection{Samples}

The following analysis is based on the data sets compiled by Schwenn et al. (2005) and Howard \& Tappin (2005). Hereinafter, we call them S-sample and $\mathrm{H}$-sample, respectively. In addition, we consulted the data sets provided by Cane \& Richardson (2003b), Zhang et al. (2003), Michałek et al. (2004), Manoharan et al. (2004), and Manoharan (2006) to cross-check the CME/ICME association in overlapping events.

The "data bank" of Schwenn et al. (2005) contains the basic data on 91 pairs of CME/ICME events in the period from January 1997 to April 2001. The starting point in forming the catalogue was to search for all CMEs that might be regarded as candidates for producing effects near the Earth, i.e., halo and partial-halo CMEs, observed by the Large Angle and Spectrometric Coronagraph (LASCO; Brueckner et al. 1995) and listed in the online LASCO-CME catalogue (http://cdaw.gsfc.nasa.gov/CME_list/; Yashiro et al. 2004). Independently, the authors searched all in-situ data measured near the Earth to identify signatures of ICMEs. The compiled "data bank" is available on the webpage at http://star.mpae.gwdg.de/cme_effects/.

The data set of Howard \& Tappin (2005) is based on measurements of 350 IP shocks (forward and reverse) recorded by the Advanced Composition Explorer (ACE; Stone et al. 1998) during the 80-month time period from January 1998 to August 2004. The data are provided in the online catalogue at http://cdsweb.u-strasbg.fr/cgi-bin/qcat?]/A+A/440/ 373. For each shock, authors identified all candidate CMEs in the LASCO CME catalogue. In most of cases there was more than one CME candidate. Among large number of parameters considered, the data set contains also the solar wind speed ahead of the shock. We employed a reduced set of 152 events, where the authors identify only a single CME candidate and where the solar wind speed was available.

\subsection{Observational parameters}

In the analysis we use the following parameters:

- TT: the Sun-Earth transit time;

- VTT: the Sun-Earth average transit speed;
- $w$ : solar wind speed at $1 \mathrm{AU}$;

- $v_{\text {proj }}$ : plane-of-sky speed of the fastest CME feature;

- $v_{\text {exp }}$ : CME “expansion" speed (S-sample only); for definition see Fig. 2 of Schwenn et al. (2005).

The transit speed VTT is calculated by dividing the distance traveled by the CME from a given position in the LASCO fieldof-view, by the corresponding time $T T$. In the following we also use the "normalized" transit time $T T^{*}$, which is the time a CME would need to traverse the distance from $R=20$ to 1 AU propagating at the velocity $V T T$. The reason for using $T T^{*}$ is to make different events comparable. In particular, we choose $R=20$ since, beyond this distance, the solar wind speed does not change much (for the slow solar wind observations, see Sheeley et al. 1997; for fast solar wind see, e.g., models by Withbroe 1988; Sittler \& Guhathakurta 1999; Mann et al. 1999; Kim et al. 2004), which simplifies the comparison with theoretical considerations.

In the case of the H-sample, for the solar wind speed we employed the values of the flow velocity ahead of the shock, provided in the original data set. In the case of the S-sample the solar wind speed is not given in the original data set, so we measured the wind speed ahead of the shock and in the wake of ICME. In all analyzed correlations we employed both values, as well as the mean of the two. It turned out that the correlation coefficients were considerably higher in the case of the mean solar wind speed, so we only present the results for this option.

In the H-sample data set, only the projected speeds $v_{\text {proj }}$ of LASCO-CMEs are provided, whereas the S-sample data set also contains the "expansion speed" $v_{\text {exp }}$, determined from the lateral expansion of the CME. In the latter case, both CME speeds were considered in the analysis, but since the analyzed correlations were much better for $v_{\exp }$ than for $v_{\text {proj }}$, in the case of S-sample we only present the results for $v_{\text {exp }}$. As demonstrated by Schwenn et al. (2005), the expansion velocity is related to the true radial CME speed $v_{\text {rad }}$; in the statistical sense, the relationship can be expressed as $v_{\text {rad }}=0.88 v_{\text {exp }}$ (see Fig. 7 of Schwenn et al. 2005).

\section{Results}

In Figs. 1a and $\mathrm{b}$ we present ICME transit times for the $\mathrm{S}$ - and $\mathrm{H}-$ samples as a function of the ICME take-off speed $v_{\text {exp }}$ and $v_{\text {proj }}$, respectively. In both samples, the transit times were estimated by recording the arrival of the ICME-driven shock, except in eight $\mathrm{S}$-sample events when no shock signature was recorded. Note that combining shock and non-shock events increases the scatter because of the offset between the shock and ICME (for the forecasting aspect see Gopalswamy et al. 2005a; for theoretical background see Russell \& Mulligan 2002). For the S-sample the average offset adds up to $7 \pm 3 \mathrm{~h}$.

The S-sample graph contains 86 events (in 5 out of 91 events $v_{\text {exp }}$ was not measured). In the $\mathrm{H}$-sample case we excluded from the initial set of 152 events 32 cases in which the estimated transit speeds VTT were very large (up to $3000 \mathrm{~km} \mathrm{~s}^{-1}$ ). Moreover, in these events the values of VTT were much higher than both the CME speed and the solar wind speed, indicating that the $\mathrm{CME} / \mathrm{ICME}$ association was probably wrong. Indeed, some of these ICMEs were related to different CMEs in some of the "control" data sets mentioned at the beginning of Sect. 2.1. The remaining set, presented in Fig. 1b, contains 120 events.

Both samples shown in Figs. 1a and b show a typical dependence of the transit time on the CME speed, reported in a number of papers (e.g., Gopalswamy et al. 2000, 2001, 2003; 

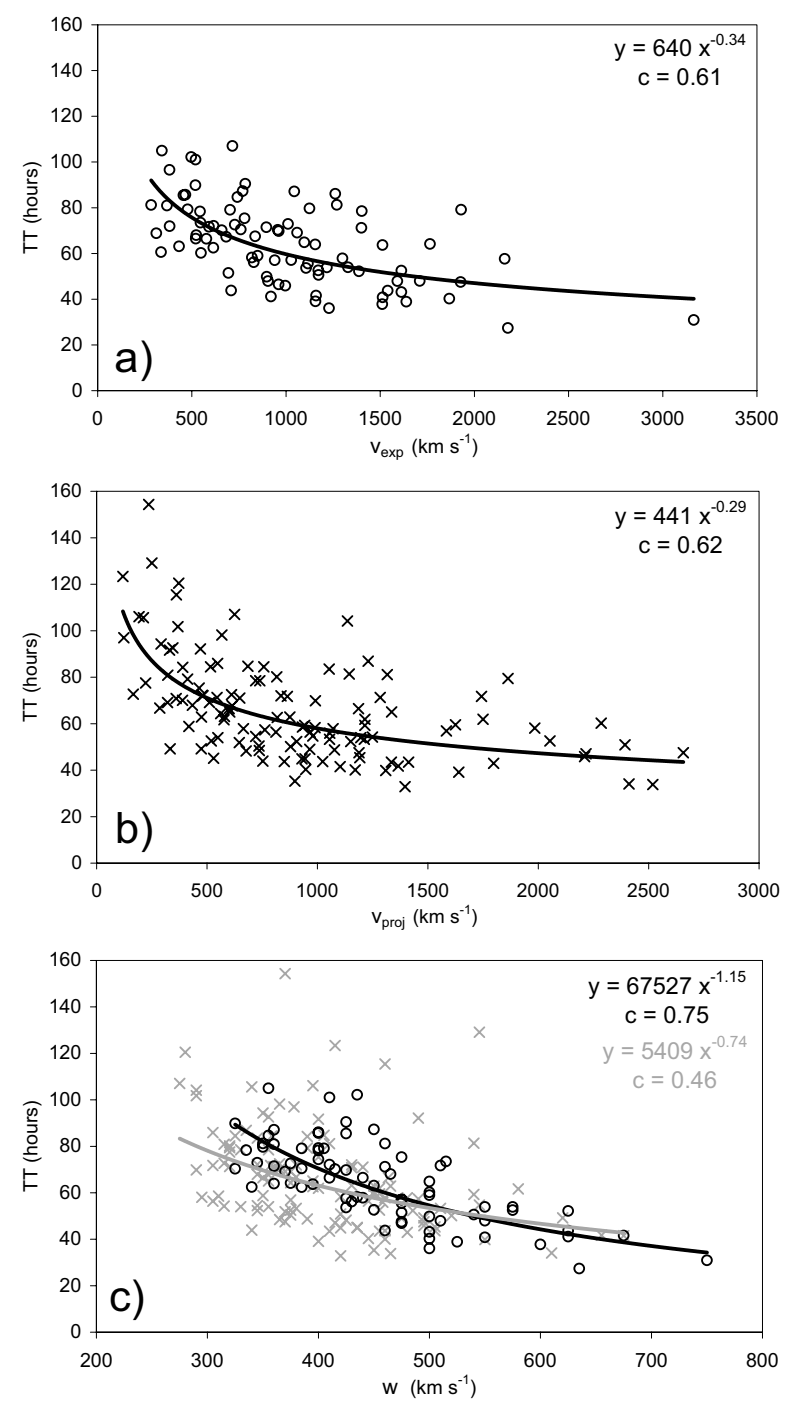

Fig. 1. ICME transit times shown as a function of a) CME expansion speed (S-sample), b) CME plane-of-sky speed (H-sample), c) solar wind velocity (S-sample - black, H-sample - gray). The power-law least-square fit parameters are given in the insets, together with the correlation coefficient $c$.

Cane \& Richardson 2003a; Cho et al. 2003; Manoharan et al. 2004; Michałek et al. 2004; Srivastava \& Venkatakrishnan 2004; Schwenn et al. 2005; Xie et al. 2006). Transit times of slow CMEs range around $100 \mathrm{~h}$, whereas they are grouped around $40 \mathrm{~h}$ in the case of the fastest CMEs. The scatter at a given $v_{\mathrm{CME}}$ is typically in the range of 30-40 h.

In Fig. 1c the transit time is presented as a function of the solar wind speed. In the S-sample case we employ the mean value of the solar wind speed measured ahead and behind the ejection. Since in 12 events we could not reliably estimate the solar wind speed (either due to data gaps, too complex a situation, or the lack of the shock), the presented sample contains 74 events. Since now the entire sample consists of shock-driving ICMEs, all $T T$ values represent the shock transit time. In the $\mathrm{H}$-sample case we use the solar wind speed measured ahead of the ejection, as reported by Howard \& Tappin (2005). Note a considerable difference between the two correlations, in particular much larger scatter in the case of $\mathrm{H}$-sample. We emphasize that similar scatter is found for the S-sample if only the solar wind speed ahead of or behind the ICME is considered. The correlation
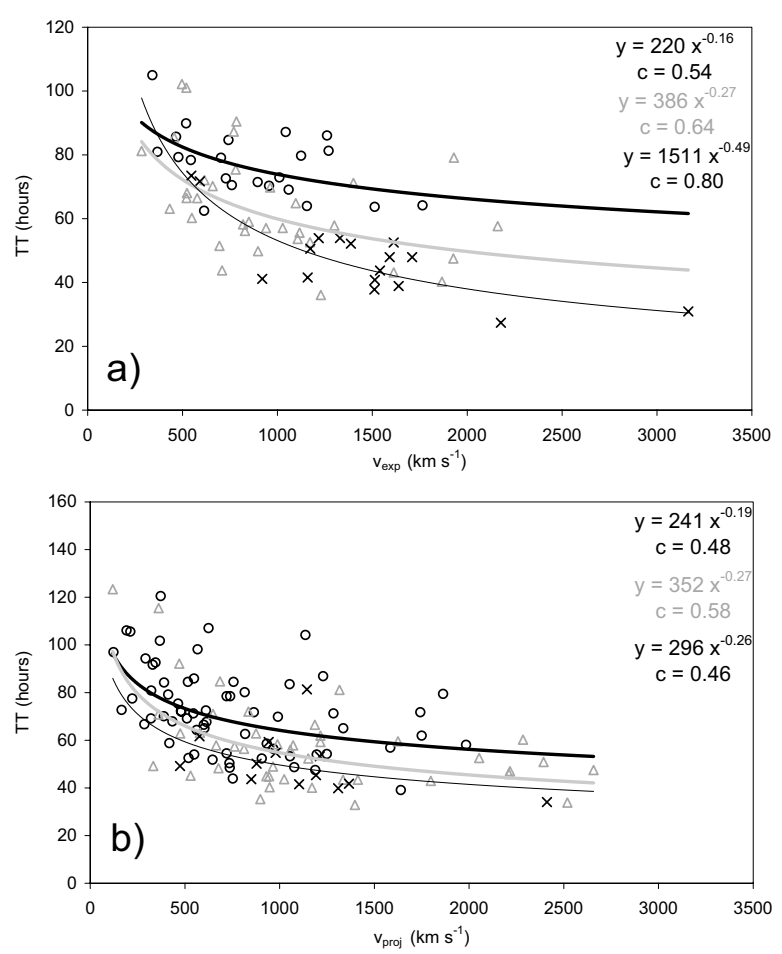

Fig. 2. CME transit times shown as a function of the CME speed: a) Ssample; b) H-sample. Data are divided into three solar wind speed bins: $w \leq 400 \mathrm{~km} \mathrm{~s}^{-1}$ (circles and black-bold line), $400<w \leq 500 \mathrm{~km} \mathrm{~s}^{-1}$ (gray triangles and gray-bold line), and $w>500 \mathrm{~km} \mathrm{~s}^{-1}$ (crosses and thin line). The power-law least-square fit parameters are shown in insets, together with the correlation coefficients $c$.

significantly improves if the mean value of the two speeds is employed.

Figure 1c clearly demonstrates that there is a large influence of the solar wind speed on the ICME transit times. Transit times associated with the slow solar wind are found in the range 80-100 h, whereas in the fast solar wind environment, transit times decrease to $30-50 \mathrm{~h}$. Bearing this result in mind, it is reasonable to assume that the data scatter present in Figs. 1a and b is to a great deal caused by differences in the solar wind speed. We note that in the S-sample case, the $T T(w)$ relationship has considerably better correlation coefficient than the relationship $T T\left(v_{\text {exp }}\right)$ shown in Fig. 1a.

To clarify to what degree the differences in the solar wind velocity contribute to the scatter of data points in Figs. 1a and b, we divided the $\mathrm{S}$ - and $\mathrm{H}$-samples into three solar wind speed bins: $w \leq 400,400<w \leq 500$, and $w>500 \mathrm{~km} \mathrm{~s}^{-1}$. The results are shown in Figs. $2 \mathrm{a}$ and $\mathrm{b}$ for the two samples, respectively. In the S-sample case we also performed the same procedure on a subset of events, where we had excluded 11 cases in which ICME transient speeds VTT were considerably greater than both the CME speed and the solar wind speed, indicating that the CME/ICME identification might be wrong (as applied in the H-sample case). The outcome for this subsample, containing 63 events, turned out to be similar to that shown in Fig. 2a, with only the correlation coefficients somewhat larger $(c=0.65,0.64$, and 0.86 for the three $w$-bins, respectively), and the fitted curves show a more distinct segregation between the three $w$-bin subsets.

Figures $2 \mathrm{a}$ and $\mathrm{b}$ clearly show that, on average, the shortest travel times are achieved by fast CMEs traveling in a fast solar wind, whereas the longest travel times are found for slow CMEs propagating in a slow solar wind. In the S-sample case, 

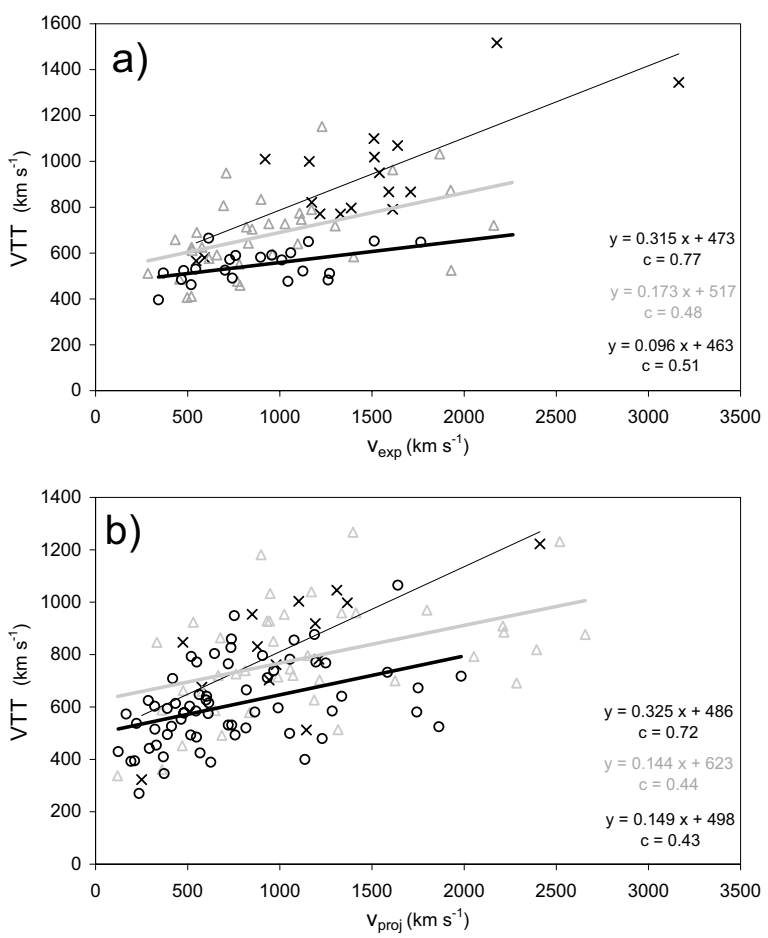

Fig. 3. Dependence of the ICME transit speed on the CME velocity: a) S-sample; b) H-sample. Data are divided into three solar wind speed bins: $w \leq 400 \mathrm{~km} \mathrm{~s}^{-1}$ (circles and black-bold line), $400<w \leq$ $500 \mathrm{~km} \mathrm{~s}^{-1}$ (gray triangles and gray-bold line), and $w>500 \mathrm{~km} \mathrm{~s}^{-1}$ (crosses and thin line). Linear least-square fit parameters are shown in insets together with the correlation coefficients $c$.

the mean transit times for the bins $w \leq 400,400<w \leq 500$, and $w>500 \mathrm{~km} \mathrm{~s}^{-1}$ are $77 \pm 10,64 \pm 16$, and $47 \pm 12 \mathrm{~h}$, respectively, whereas in the H-sample case they equal $72 \pm 18,58 \pm 19$, and $50 \pm 12$ h. Inspecting the fitted curves in Figs. $2 \mathrm{a}$ and $\mathrm{b}$, we again find that the transit times at a given CME speed are shorter for about 20-30 $\mathrm{h}$ in the case of a fast solar wind than for a slow solar wind. Yet, it should be noted that the scatter of data-points cannot be attributed solely to differences in solar wind speed, since a relatively large scatter is still present in each $w$-bin (especially in the bin $400<w \leq 500 \mathrm{~km} \mathrm{~s}^{-1}$ ), though somewhat smaller than what is present in Fig. 1a.

Another useful parameter in analyzing the propagation of ICMEs is the transit speed $V T T$, defined as the ratio of the distance traveled by ICME and the corresponding transit time $T T$. In Fig. 3 we show the transit speeds as a function of the CME speed, dividing the data into the same three solar wind speed bins as in Fig. 2. In the S-sample case (Fig. 3a) for the CME speed we use $v_{\text {exp }}$, and in the case of H-sample (Fig. 3b) we use $v_{\text {proj. }}$. Again, like in Fig. 2, the data scatter is somewhat smaller in the S-sample case than in the H-sample case. Similarly, the correlation in the $\mathrm{S}$-sample is more coherent for $v_{\text {exp }}$ compared to the option with $v_{\text {proj }}$ (not shown). This indicates that $v_{\exp }$ is better parameter in forecasting the ICME arrival time, than $v_{\text {proj }}$.

The advantage of using VTT is twofold. First, the $V T T\left(v_{\mathrm{CME}}\right)$ relationship can be naturally fitted by the linear dependence. Second, it provides a "normalization" of all transit times to any chosen initial radial distance, common to all CMEs (note that changes in the CME velocity in the LASCO field of view can introduce a certain error, but it is negligible compared to other sources of errors). The second aspect is also important from the theoretical point of view, since the initial
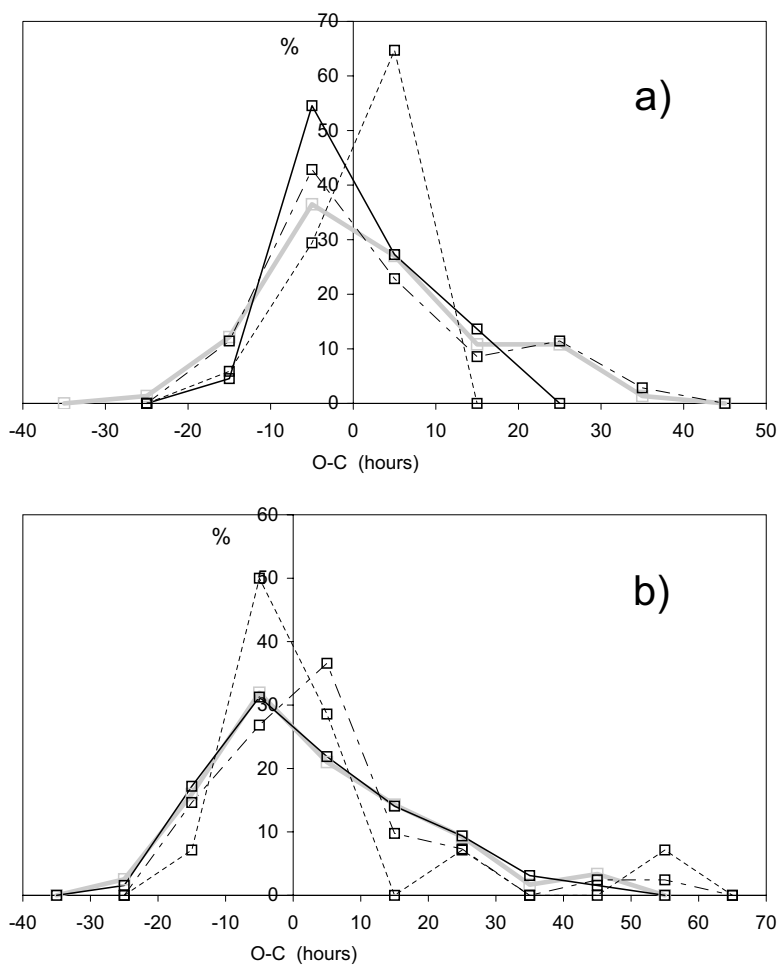

Fig. 4. The percentage distribution of $\mathrm{O}-\mathrm{C}$ values in 10-hours bins: a) S-sample; b) H-sample. The solar wind bins $w \leq 400,400<w \leq 500$, and $w>500 \mathrm{~km} \mathrm{~s}^{-1}$ are represented by the thin, dash-dotted, and dotted lines, respectively. The distribution concerning the complete sample with neglected differences in the solar wind is shown by a bold-gray line.

distance can be set to a relatively large height (e.g., the outer edge of the LASCO-C3 field-of-view), beyond which the solar wind can be taken as approximately constant (see, e.g., Sheleey et al. 1997), and the Lorentz force becomes small (see e.g., Vršnak et al. 2004a). Both effects simplify the theoretical considerations (see, e.g., Cargill 2004).

\section{Summary of correlations}

In Table 1 we summarize the characteristics of the analyzed correlations. In the first two columns we label the sample and show the number of the events involved. In the next two columns we present the linear and power-law least-square fit parameters for the $\operatorname{VTT}\left(v_{\mathrm{CME}}\right)$ and $T T^{*}\left(v_{\mathrm{CME}}\right)$ correlation, together with the correlation coefficient $c$. The $T T^{*}$ values correspond to the transit time from the radial distance of 20 solar radii to $1 \mathrm{AU}$. The statistical significance of all these correlations is greater than $99 \%$, meaning that the probability for an accidental distribution of data points is less than $1 \%$.

To quantify the scatter of the data, we employed the parameter $\mathrm{O}-\mathrm{C}$, defined as the difference between the observed transit times and the values calculated by substituting $v_{\mathrm{CME}}$ into the corresponding power-law least-square fit $T T\left(v_{\mathrm{CME}}\right)$. We applied this procedure to $T T$, as well as to $T T^{*}$ values, using the power-law least-square fit parameters displayed in Table 1 and Figs. 1, 2, respectively. We found only a minor, statistically insignificant difference, where $\mathrm{O}-\mathrm{C}$ values turned out to be somewhat lower for $T T^{*}$ than for $T T$. In Figs. 4a and b we show the distribution of $\mathrm{O}-\mathrm{C}$ values regarding the values $T T^{*}$ for all events in $\mathrm{S}$ - and H-samples, where the effect of the solar wind speed is neglected, i.e., the power-law fit displayed in the first row of 
Table 1. Summary of correlations.

\begin{tabular}{|c|c|c|c|c|c|c|}
\hline sample & $N$ & $V T T\left(v_{\mathrm{CME}}\right)$ & $T T^{*}\left(v_{\mathrm{CME}}\right)$ & $\%_{10 h}$ & $\overline{\delta_{\%}}$ & $\mathrm{rms}$ \\
\hline $\mathrm{S}$ (all) & 74 & $V T T=(0.28 \pm 0.04) v_{\exp }+(405 \pm 43) ; c=0.67$ & $T T^{*}=738 v_{\exp }^{-0.38 \pm 0.05} ; c=0.65$ & $63 \%$ & $17 \%$ & 12.2 \\
\hline $\mathrm{S}(w \leq 400)$ & 22 & $V T T=(0.10 \pm 0.04) v_{\exp }+(463 \pm 34) ; c=0.51$ & $T T^{*}=200 v_{\exp }^{-0.16 \pm 0.05} ; c=0.54$ & $82 \%$ & $13 \%$ & 8.0 \\
\hline$S(400<w \leq 500)$ & 35 & $V T T=(0.17 \pm 0.06) v_{\exp }+(517 \pm 59) ; c=0.48$ & $T T^{*}=354 v_{\exp }^{-0.27 \pm 0.07} ; c=0.52$ & $66 \%$ & $17 \%$ & 12.8 \\
\hline $\mathrm{S}(w>500)$ & 17 & $V T T=(0.31 \pm 0.067) v_{\exp }+(473 \pm 105) ; c=0.77$ & $T T^{*}=1374 v_{\exp }^{-0.48 \pm 0.09} ; c=0.80$ & $94 \%$ & $9 \%$ & 6.4 \\
\hline $\mathrm{H}($ all $)$ & 120 & $V T T=(0.20 \pm 0.03) v_{\text {proj }}+(511 \pm 31) ; c=0.54$ & $T T^{*}=395 v_{\text {proj }}^{-0.29 \pm 0.03} ; c=0.62$ & $49 \%$ & $22 \%$ & 17.1 \\
\hline $\mathrm{H}(w \leq 400)$ & 65 & $V T T=(0.15 \pm 0.04) v_{\text {proj }}+(498 \pm 35) ; c=0.42$ & $T T^{*}=151 v_{\text {proj }}^{-0.21 \pm 0.05} ; c=0.51$ & $53 \%$ & $20 \%$ & 16.8 \\
\hline $\mathrm{H}(400<w \leq 500)$ & 41 & $V T T=(0.14 \pm 0.05) v_{\text {proj }}+(623 \pm 62) ; c=0.44$ & $T T^{*}=321 v_{\text {proj }}^{-0.27 \pm 0.06} ; c=0.59$ & $63 \%$ & $22 \%$ & 15.7 \\
\hline $\mathrm{H}(w>500)$ & 14 & $V T T=(0.32 \pm 0.09) v_{\text {proj }}+(486 \pm 105) ; c=0.72$ & $T T^{*}=1153 v_{\text {proj }}^{-0.47 \pm 0.12} ; c=0.75$ & $79 \%$ & $19 \%$ & 16.7 \\
\hline
\end{tabular}

Table 1 is utilized. Furthermore, we present the analogous $w$ bin based distributions, evaluated by applying the corresponding $w$-bin power-law fits specified in Table 1 .

The basic characteristics of $\mathrm{O}-\mathrm{C}$ distributions are presented in the last three columns of Table 1: in the fifth column we present the percentage of events for which the difference between the observed and calculated transit times $|\mathrm{O}-\mathrm{C}|$ is less than $10 \mathrm{~h}$; the mean value, $\bar{\delta}_{\%}$, of the relative difference $\delta_{\%}=$ $100 \times|\mathrm{O}-\mathrm{C}| / \mathrm{C}$ is presented in the sixth column; in the last column we present the root-mean-square (rms) values, expressed in h. Note that we prefer to use the form $(\mathrm{O}-\mathrm{C}) / \mathrm{C}$ instead of the more common form $(\mathrm{O}-\mathrm{C}) / \mathrm{O}$, since only the $\mathrm{C}$-value is known in the forecasting situation. Consequently, to predict how much the observed value might deviate from the calculated one, we have to know a typical deviation expressed in terms of $\mathrm{C}$. In this respect let us note that $(\mathrm{O}-\mathrm{C}) / \mathrm{C}$ and $(\mathrm{O}-\mathrm{C}) / \mathrm{O}$ values on average do not differ significantly.

Inspecting the data in the last three columns of Table 1, i.e., comparing row 1 with rows 2,3 , and 4 , and row 5 with rows 6 , 7 , and 8 , we find that the data based on $w$-bins generally provide a considerably better $\mathrm{O}-\mathrm{C}$ outcome. This implies that the prediction of ICME arrival times could be improved by taking the solar wind speed into account.

Since the transit times (and thus the transit speed) depend on both the CME speed and the solar wind velocity, we also performed a two-parameter linear least-square fit of the form $V T T=k_{0}+k_{v} v_{\mathrm{CME}}+k_{w} w$. The correlation parameters for S-sample read $k_{0}=-111 \pm 75 \mathrm{~km} \mathrm{~s}^{-1}, k_{1}=0.16 \pm 0.03$, and $k_{2}=1.41 \pm 0.18$. After calculating the $T T^{*}$ values based on this correlation, one finds that the percentage of events that fall into the range $|\mathrm{O}-\mathrm{C}|<10 \mathrm{~h}$ amounts to $76 \%$, which is considerably more than $63 \%$, which in turn is found without taking the solar wind speed into account (i.e., based on the correlation presented in the first row of Table 1). For the reduced $S$-sample containing 63 events (see Sect. 3), one finds the increase from $67 \%$ to $78 \%$, whereas in the case of $\mathrm{H}$-sample, the the percentage increases from $49 \%$ to $59 \%$. This demonstrates that the $\mathrm{O}-\mathrm{C}$ outcome is considerably better if the effect of the solar wind speed is taken into account, as we already found applying the $w$-bin procedure (last three columns of Table 1).

\section{Discussion and conclusion}

We summarize the results of the analysis as follows:

- ICME transit times depend on the ICME take-off speed, as well as on the solar wind speed. The transit times of ICMEs that are associated with solar wind faster than $500 \mathrm{~km} \mathrm{~s}^{-1}$, on average are shorter by $20-30 \mathrm{~h}$, i.e., around 1 day, than those associated with the wind slower than $400 \mathrm{~km} \mathrm{~s}^{-1}$.

- The relationship between the transit times and the solar wind speed is more coherent if the mean value of the wind speed ahead of and behind the ICME is considered than if only one of these two values is taken into account.

- If the effect of different solar wind speeds is taken into account, the $\mathrm{O}-\mathrm{C}$ values decrease - for the S-sample we find the arrival times in the $\mathrm{O}-\mathrm{C}$ frame of $\pm 10 \mathrm{~h}$ in about $80 \%$ of ICMEs; in the case of fast CMEs, the percentage is even $>90 \%$.

- The two-parameter linear least-square fit of the form VTT = $k_{0}+k_{v} v_{\mathrm{CME}}+k_{w} w$ gives for the S-sample the coefficients $k_{0}=-111 \pm 75 \mathrm{~km} \mathrm{~s}^{-1}, k_{1}=0.16 \pm 0.03$, and $k_{2}=1.41 \pm 0.18$.

The situation where the ICME propagates through a high-speed solar wind stream arises when the CME source region is near to, or bracketed by, equatorial coronal holes; for the relationship between the coronal hole area/position and characteristics of the associated fast streams, see Robbins et al. (2006) and Vršnak et al. (2007b), and references therein. This has an element of practicality because CMEs originating from the neighborhood of coronal holes are likely to have shorter transit times.

The presented results can be compared quantitatively with expectations based on a simple kinematical model, where all forces except the "aerodynamic" drag are neglected. In such a case the equation of motion reads

$a=-\gamma(v-w)|v-w|$,

where $a$ and $v$ are the acceleration and velocity of ICME and $w$ is the solar wind speed. The parameter $\gamma$ can be expressed as $\gamma=c_{\mathrm{d}} A \rho_{w} / M$, where $A$ is the area of the ICME cross-section, $\rho_{w}$ the ambient density, $M$ the mass of ICME, whereas $c_{\mathrm{d}}$ represents the dimensionless drag coefficient (for details see Cargill 2004).

For the sake of simplicity, we assume that the mass of ICME remains constant, and we consider the motion beyond the distance of 20 solar radii, where the solar wind speed becomes practically constant and the density decreases with the distance as $\rho_{w} \approx R^{-2}$ (see, e.g., the empirical models by Sheeley et al. 1997; Leblanc et al. 1998; Vršnak et al. 2004b, or the basic theoretical models by Parker 1958; Mann et al. 1999). A more detailed consideration of the kinematical modeling will be presented in a separate paper.

Assuming that the dimensions of ICMEs are roughly proportional to the heliocentric distance, i.e., $A \propto R^{2}$, and taking into account that $\rho_{w} \approx R^{-2}$, one finds $\gamma \approx$ const., since generally, $c_{\mathrm{d}}$ should not change much with the distance (for details see Cargill 2004). In Fig. 5a we present the calculated 

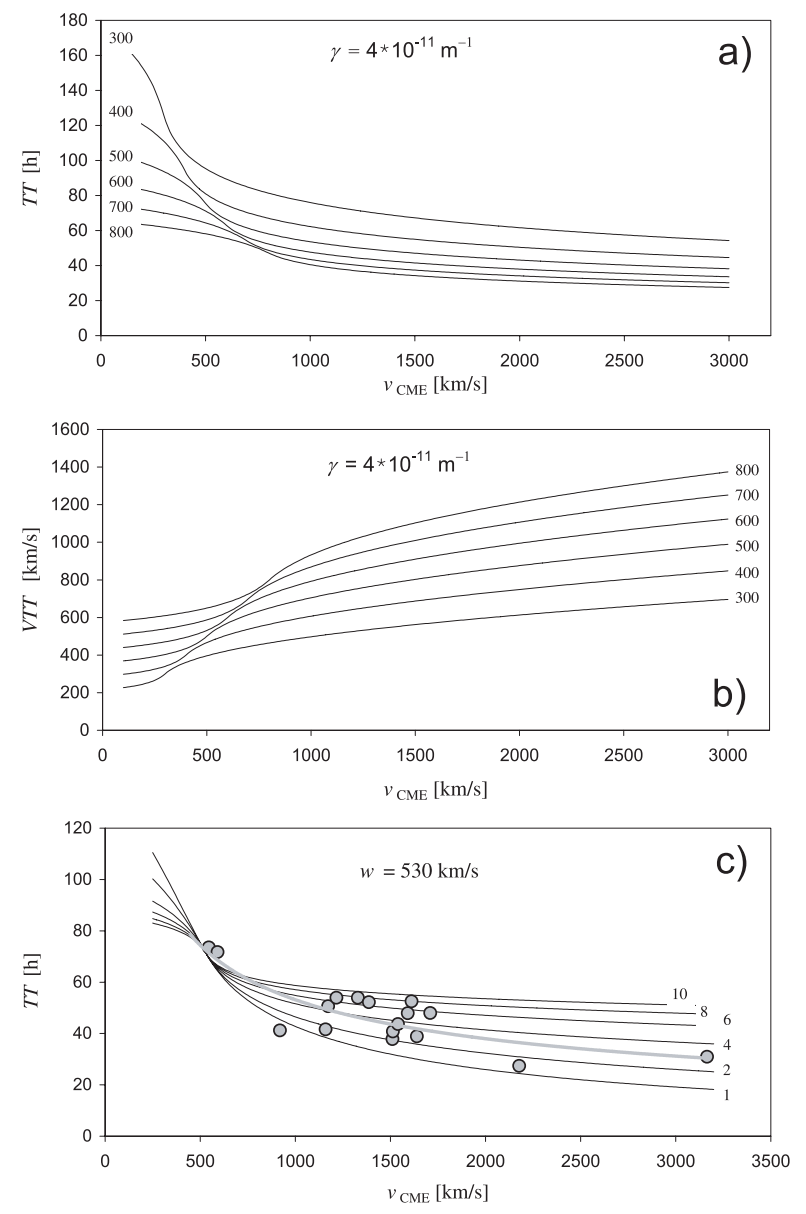

Fig. 5. a) Transit times calculated using $\gamma=4 \times 10^{-11} \mathrm{~m}^{-1}$ and the solar wind speed in the range $w=300-800 \mathrm{~km} \mathrm{~s}^{-1}$ (the values of $w$ are indicated by the curves). b) Transit speeds calculated for the same set of parameters. c) Transit times calculated using $w=530 \mathrm{~km} \mathrm{~s}^{-1}$ and the values of $\gamma$ in the range $10^{-11}-10^{-10} \mathrm{~m}^{-1}$ (the values of $\gamma$ expressed in $10^{-11} \mathrm{~m}^{-1}$ are written by the curves). The model values are compared with the measured data in the bin $w>500 \mathrm{~km} \mathrm{~s}^{-1}$.

transit times from $R=20$ as a function of the take-off speed, calculated for different solar wind speeds and using $\gamma=4 \times$ $10^{-11} \mathrm{~m}^{-1}$. In Fig. $5 \mathrm{~b}$ the corresponding transit speeds are presented. Comparing Fig. 5a with Fig. 2 and Fig. 5b with Fig. 3, one finds a good correspondence; i.e., even the simplest dragbased kinematical model can explain basic features of the observations.

In Fig. 5c we compare the observed transit times in the bin $w>500 \mathrm{~km} \mathrm{~s}^{-1}$, with model values calculated using $w=$ $530 \mathrm{~km} \mathrm{~s}^{-1}$, which is the mean of the solar wind speeds in this bin. We used values of $\gamma$ in the range $10^{-11}-10^{-10} \mathrm{~m}^{-1}$ to account for different ICME masses/densities and different solar wind densities. The outcome illustrates that the scatter of the data in a given $w$-bin is probably not only due to ambiguities in determining $v_{\mathrm{CME}}$ or $w$, but is also caused by different ICME masses or other factors that determine the characteristics of the drag through the parameter $\gamma$.

Recently, Robbins et al. (2006) and Vršnak et al. (2007b) proposed a method of forecasting the 1 AU solar wind speed from measurements of the position and area of coronal holes in periods of low solar activity. In such periods, the forecasted solar wind speed could be used in combination with the CME take-off speed to predict the ICME transit time, employing the correlations we established in this paper, so our results could be applied to improve statistical forecasting of the ICME arrivals. However, it should be noted that, on average, the accuracy of predicted arrivals cannot be better than $\pm 10 \mathrm{~h}$ (Fig. 5c).

In principle, the method proposed by Vršnak et al. (2007b) could be extended to map the solar wind characteristics in the ecliptic plane. This could be used to gain insight into the characteristics of the solar wind an ICME would "see" on its way to Earth. In other words, one could get a rough estimate of the dependence $w(R)$ along the ICME trajectory. Potentially, that would improve the kinematical modeling based on Eq. (1), where the comparison of the model curves like those shown in Fig. 5 with the empirical data shown in Figs. 2 and 3, would give an insight into the characteristics of the drag parameter $\gamma$. However, it should be noted that a more reliable forecast based on kinematical modeling (but also in the case of the full MHD modeling) could be performed only if the difference in $\mathrm{CME}$ masses/densities is taken into account.

Finally, let us comment some interesting side results of our analysis. The first one concerns a relatively small number of ICMEs associated with the solar wind high-speed streams (HSSs) - from the 2nd column of Table 1 we find $23 \%$ of such events in the S-sample and $12 \%$ in the $\mathrm{H}$-sample (see also Fig. 1c). Such a low percentage could be a consequence of two effects. First, it may indicate that CMEs are generally launched from regions far from coronal holes. On the other hand, our data set includes only shock-driving ICMEs, which means that they must be fast if they propagate through the high-speed solar wind, i.e., slow CMEs will be a priori excluded, reducing the number of HSS-associated ICMEs in our sample.

Another interesting detail concerns the relatively large number of slow CMEs $\left(v<500 \mathrm{~km} \mathrm{~s}^{-1}\right)$, particularly in the H-sample (Fig. 1a). Although originating from CMEs of low plane-of-sky speed, ICMEs were all recognized as shock-driving ICMEs. This apparently surprising situation is probably related to projection effects, i.e., the true velocities were probably higher (Vršnak et al. 2007a), since most of the events were launched from close to the solar disc center. Furthermore, a fraction of the slow events might be related to gradually accelerating CMEs, whose velocity in the coronagraphic field of view is permanently increasing, so although the mean velocity is relatively low, the speed at greater heights could be considerably higher.

Our last point is related to the asymptotic trend of the fitted function $T T\left(v_{\mathrm{CME}}\right)$ at high velocities. In this range, the $T T\left(v_{\mathrm{CME}}\right)$ curves presented in Figs. 1 and 2 attain values around 30-40 h. This could give a false impression that even the fastest possible CMEs need at least $\approx 1.5$ days to arrive on Earth. However, bearing the overall data scatter in mind and relatively small number of events in this range, one could presume that the fastest CMEs could arrive in less than one day. Indeed, such events are observed - the shortest recorded transit times are shorter than 20 h (see Fig. 8 in Gopalswamy et al. 2005b). Note that the transit speed of the ICME that arrives on Earth in less than $20 \mathrm{~h}$ has to be faster than $2000 \mathrm{~km} \mathrm{~s}^{-1}$, whereas according to Eq. (1) in Gopalswamy et al. (2005b), the coronagraphic CME speed corresponding to $T T=20 \mathrm{~h}$ has to be $2100 \mathrm{~km} \mathrm{~s}^{-1}$. Such a high non-decelerated speed could be maintained through the interplanetary space only if the CME is very massive and the propelling force acts over large distances, so that the ICME is not decelerated much by the drag force. Note that at the highest CME speeds, exceeding $3500 \mathrm{~km} \mathrm{~s}^{-1}$, the transit times could approach $\approx 1 / 2$ days (Gopalswamy et al. 2005b).

Acknowledgements. This work is sponsored by the Air Force Office of Scientific Research, USAF, under grant number FA8655-06-1-3036. We are thankful to the 
ACE, Wind-Waves, LASCO-SOHO, and GOES teams for operating the instruments and performing the basic data reduction. We are especially grateful to Nat Gopalswamy, Seiji Yashiro, Grzegorz Michałek and their colleagues for compiling the online LASCO CME Catalog, as well as to the authors of the papers Schwenn et al. (2005) and Howard \& Tappin (2005) for providing the online data for their CME/ICME samples. We appreciate constructive comments and suggestions by the referee, Nat Gopalswamy, which led to significant improvement in the paper.

\section{References}

Bothmer, V., \& Schwenn, R. 1995, J. Geomagn. Geoelectr., 47, 1127 Brueckner, G. E., Howard, R. A., Koomen, M. J., et al. 1995, Sol. Phys., 162, 357

Cane, H. V., \& Richardson, I. G. 2003a, Geophys. Res. Lett., 24, 2233

Cane, H. V., \& Richardson, I. G. 2003b, J. Geophys. Res., 108, 1156

Cho, K.-S., Moon, Y.-J., Dryer, M., Fry, C. D., Park, Y.-D., \& Kim, K.-S. 2003, J. Geophys. Res., 108(A12), 1445

Cargill, P. J. 2004, Sol. Phys., 221, 135

Dyer, M., Smith, Z., Fry, C. D., et al. 2004, Space Weather, 2, S09001

Fry, C. D., Dryer, M., Deehr, C. S., et al. 2003, J. Geophys. Res., 108, 1070

Gopalswamy, N., Lara, A., Lepping, R. P., et al. 2000 Geophys. Res. Lett., 27, 145

Gopalswamy, N., Lara, A., Yashiro, S., Kaiser, M. L., \& Howard, R. A. 2001 J. Geophys. Res., 106, 29207

Gopalswamy, N., Manoharan, P. K., \& Yashiro, S. 2003, Geophys. Res. Lett., 30 Gopalswamy, N., Lara, A., Manoharan, P. K., \& Howard, R. A. 2005a, Adv. Space Res., 36, 2289

Gopalswamy, N., Yashiro, S., \& Liu, Y., et al. 2005b, J. Geophys. Res., 110

Gopalswamy, N. 2006, Space Sci. Rev., 124, 145

Gosling, J. T., Bame, S. J., McComas, D. J., \& Phillips, J. L. 1990, Geophys. Res. Lett., 17, 901

Howard, T. A, \& Tappin, S. J. 2005, A\&A, 440, 373

Kim, S.-J., Kim, K.-S., Moon, Y.-J., Cho, K.-S., \& Park, Y. D. 2004, J. Korean Astr. Soc. 37, 55

Koskinen, H. E. J., \& Huttunen, K. E. J. 2006, Space Sci. Rev., 124, 169
Leblanc, Y., Dulk, G. A., \& Bougeret, J.-L. 1998, Sol. Phys., 183, 165

Manchester, W. B., Gombosi, T. I., Russev, I., et al. 2004, J. Geophys. Res., 109, A01102

Mann, G., Jansen, F., MacDowall, R. J., Kaiser, M. L., \& Stone, R. G. 1999, A\&A, 348, 614

Manoharan, P. K., Gopalswamy, N., Yashiro, S., Lara, A., Michałek, G., \& Howard, R. A. 2004, J. Geophys. Res., 109, A06109

Manoharan, P. K. 2006, Sol. Phys., 235, 345

McKenna-Lawlor, S. M. P., Dryer, M., Kartalev, M. D., et al. 2006, J. Geophys. Res., 111, A11103

Michałek, G., Gopalswamy, N., \& Yashiro, S. 2003, ApJ, 584, 472

Michałek, G., Gopalswamy, N., Lara, A., \& Manoharan, P. K. 2004, A\&A, 423, 729

Parker, E. N. 1958, ApJ, 128, 664

Russell, C. T., \& Mulligan, T. 2002, Planet. Space Sci., 50, 527

Odstrčil, D., Pizzo, V. J., \& Arge, C. N. 2005, J. Geophys. Res., 110, A02106

Oler, C. 2004, Space Weather, 2, S08001

Robbins, S., Henney, C. J., \& Harvey, J. W. 2006, Sol. Phys., 233, 265

Schwenn, R., Dal Lago, A., Huttunen, E., \& Gonzalez, W. D. 2005, Annales Geophys. 23, 1033

Sittler, E. C. Jr., \& Guhathakurta, M. 1999, ApJ, 523, 812

Sheeley, N. R. Jr., Wang, Y.-M., Hawley, S. H., et al. 1997, ApJ, 484, 472

Srivastava, N., \& Venkatakrishnan, P. 2004, J. Geophys. Res., 109, A10103

Stone, E. C., Frandsen, A. M., Mewaldt, R. A., et al. 1998, Space Sci. Rev., 86, 1

Tsurutani, B. T., \& Gonzalez, W. D. 1998, in Magnetic Storms, ed. B. T. Tsurutani, et al., Geophys. Monogr. 98 (Washington, DC: AGU), 77

Vršnak, B. 2001, Sol. Phys., 202, 173

Vršnak, B., \& Gopalswamy, N. 2002, J. Geophys. Res., 107(A2), 1019

Vršnak, B., Ruždjak, D., Sudar, D., \& Gopalswamy, N. 2004a, A\&A, 423, 717

Vršnak, B., Magdalenić, J., \& Zlobec, P. 2004b, A\&A, 413, 753

Vršnak, B., Temmer, M., \& Veronig, A. M. 2007b, Sol. Phys., 240, 315

Vršnak, B., Sudar, D., Ruždjak, D., \& Žic, T. 2007a, A\&A, 469, 339

Withbroe, G. L. 1988, ApJ, 325, 442

Xie, H., Offman, L., \& Lawrence, G. 2004, J. Geophys. Res.

Xie, J., Wei, F., Xiang, C., \& Feng, X. 2006, Sol. Phys., 238, 377

Yashiro, S., Gopalswamy, N., Michałek, G., et al. 2004, J. Geophys. Res., 109

Zhang, J., Dere, K. P., Howard, R. A., \& Bothmer, V. 2003, ApJ, 582, 520 